\title{
L'espace minéral au Paléolithique moyen dans les départements du Cantal, de la Haute-Loire et du Puy-de-Dôme
}

Jean-Paul Raynal, Paul Fernandes et Michel Piboule

\section{Q OpenEdition}

\section{Journals}

Édition électronique

URL : http://journals.openedition.org/adlfi/4919

ISSN : 2114-0502

Éditeur

Ministère de la culture

Référence électronique

Jean-Paul Raynal, Paul Fernandes et Michel Piboule, "L'espace minéral au Paléolithique moyen dans les départements du Cantal, de la Haute-Loire et du Puy-de-Dôme », ADLFI. Archéologie de la France Informations [En ligne], Auvergne, mis en ligne le 01 mars 2006, consulté le 01 mai 2019. URL : http:// journals.openedition.org/adlfi/4919

Ce document a été généré automatiquement le 1 mai 2019.

(c) Ministère de la Culture et de la Communication, CNRS 


\title{
L'espace minéral au Paléolithique moyen dans les départements du Cantal, de la Haute-Loire et du Puy- de-Dôme
}

\author{
Jean-Paul Raynal, Paul Fernandes et Michel Piboule
}

Date de l'opération : 2006 (PT)

Les travaux se sont déroulés de juillet à la fin novembre 2006 dans les départements de la Haute-Loire, du Cantal et du Puy-de-Dôme et ont été complétés par des travaux dans l'Allier, suite aux prospections antérieures de l'un d'entre nous (Maurice Piboule).

2 Notre démarche n'est pas un simple complément aux nombreuses études régionales consacrées au silex depuis Vinay en 1867 : aucune démarche antérieure n'a en effet utilisé une méthodologie minéralogique et pétrographique compatible avec nos exigences de collecte de données.

3 Rappelons brièvement que seule une étude complète des échantillons représentatifs aux trois échelles (macroscopique, microscopique, ultramicroscopique) permet de décrypter les effets des agents mécaniques et physicochimiques inscrits sur la surface et dans les zones endocorticales et internes des matériaux. Les stigmates les plus tardifs ainsi acquis à la suite de la mise à l'air libre du silex sont de bons indicateurs de son évolution, de ses lieux de résidence et de son site final de collecte. Cette démarche permet de constituer une série de référentiels précis, complémentaires aux seules déterminations pétrographiques ou chimiques proposées jusqu'à maintenant et le plus souvent pour les seuls gîtes primaires : pour le préhistorien, connaître les matériaux à tous les stades de leurs chaînes évolutives (types génétiques, types gîtologiques et types archéologiques) apparaît indispensable pour cerner utilement leurs origines géologiques et géographiques.

4 Les objectifs 
5 - Affiner la vision des ressources disponibles dans le proche environnement des sites du Paléolithique moyen de Sainte-Anne 1, Rochelimagne et Le Ronddu Barry (niveau H) à Polignac, Baume-Vallée à Solignac-sur-Loire, le Rond de Saint-Arcons-d'Allier, et caractériser les corpus d'artéfacts dans les milieux géochimiques ouverts à Saint-PierreEynac, Tronquière à Aurillac, Salièges à Montmurat.

6 - Vérifier l'existence de silex jurassiques et crétacés en position secondaire au centre du massif, définir le corpus des silex géologiquement exogènes.

7 - Prélever des échantillons en place dans les formations lacustres des différents bassins tertiaires, en notant leur polarité, leur position dans la formation, leur taille et leur morphologie.

8 - Collecter des échantillons à des distances variables du gite primaire en fonction du contexte géologique et géomorphologique, selon les différents axes de dispersion (dans chaque bassin sédimentaire), en respectant leur polarité, leur position et en notant la taille, la variabilité et la richesse pour chaque gîte secondaire, dans le but de cataloguer les contrastes génomorphiques et phénomorphiques propres à l'évolution des silex.

9 - Prélever des échantillons en place dans les silicifications pédologiques et hydrothermales, en notant leur polarité, leur position dans l'encaissant, leur taille et leur morphologie.

\section{Principaux résultats obtenus}

11 - Remise en cause de la pauvreté en sources :

12 la notion de pauvreté régionale en matières premières est toute relative, la quantité des gîtes actuellement inventoriés en est une preuve évidente.

13 -Typologie des silex et cherts rhétiens et jurassiques mieux définie :

14 ce travail a dans un premier temps permis de déterminer les types génétiques présents dans les alluvions anciennes du Massif central et pose le problème de la variété des sources. Ces silex et cherts marins semblent provenir des bordures ardéchoises et lozériennes.

15 - Typologie des silex des bassins oligocènes encours d'élaboration :

16 même si les séries carbonatées offrent une grande diversité de faciès, nous sommes désormais capables de distinguer les silex des cinq bassins les plus remarquables du Massif central : le bassin de Maurs (Ludien), le bassin du Puy-en-Velay (Sannoisien), le bassin du Malzieu (Sannoisien), le bassin de Saint-Alban (Stampien) et le bassin d'Aurillac (Stampien).

17 -Typologie des silex des différentes formations miocènes :

18 il n'est plus possible d'affirmer que tous les silex auvergnats sont associés aux séries carbonatées. Ce travail nous a conduit à préciser la grande variabilité des silicifications miocènes en position primaire et secondaire et leur importance en tant que ressources utilisées par les hommes du Paléolithique moyen.

19 - Remise en cause de l'absence des silex crétacés :

20 il existe des silex crétacés en position naturelle en Auvergne, qui ont transité dans le domaine régionale à partir de formations anciennes comme celles de Naussac (Lozère).Ces silex marins semblent provenir des formations du Crétacé inférieur de la bordure sud-est du Massif central. Les sources les plus proches sont à l'est, les plateaux ardéchois, à l'ouest la zone de Cahors. 

l'Éocène. Cet inventaire des gîtes permet de diversifier les origines de ces matériaux sur la base de leurs précurseurs (protolites) et d'établir des faciès génétiques qui leur sont spécifiques. Au plan archéologique, cette reconnaissance pétrogénétique permet d'affiner l'origine d'une partie des sources de matières siliceuses des industries moustériennes dans une région où les silicifications cénozoïques sont fréquentes. des «chailles» est confirmée. La nature polygénique des matériaux siliceux de cette formation pose le problème de l'origine géographique des sources alimentant les alluvions de la paléo-Loire et de ses affluents.

27 RAYNAL Jean-Paul, FERNANDES Paul et PIBOULE Michel

\section{INDEX}

Index géographique : Auvergne

operation Prospection thématique (PRT)

Thèmes : géomorphologie, inventaire, méthodologie, silex, typologie, typologie lithique

Index chronologique : Paléolithique moyen

\section{AUTEURS}

JEAN-PAUL RAYNAL

CNRS 